\title{
Revisited Doniach diagram: Influence of short-range antiferromagnetic correlations in the Kondo lattice
}

\author{
J. R. Iglesias \\ Instituto de Física, Universidade Federal do Rio Grande do Sul, CP 15051, 91501 - 970 Porto Alegre, Brazil \\ C. Lacroix
}

Laboratoire L. Néel, CNRS, B. P. 166, 38042 Grenoble Cedex 09, France

B. Coqblin

Laboratoire de Physique des Solides, Bât. 510, Université Paris-Sud, 91405 Orsay, France

(Received 5 June 1997)

\begin{abstract}
The Kondo lattice model including nearest-neighbor magnetic exchange interactions is studied here in a mean-field approximation describing both the Kondo state and the intersite magnetic correlations. In the case of antiferromagnetic correlations, our model yields a decrease of the Kondo temperature compared to the one-impurity value, giving, therefore, a "revisited" version of the Doniach diagram with a rather flat Kondo temperature in the nonmagnetic case, as observed in several cerium compounds. Our model shows also that short-range magnetic correlations can appear at a temperature clearly larger than the Kondo temperature, as observed in compounds such as $\mathrm{CeCu}_{6}$ or $\mathrm{CeRu}_{2} \mathrm{Si}_{2}$. [S0163-1829(97)04642-0]
\end{abstract}

\section{INTRODUCTION}

It is well known that the one-impurity Kondo effect is described by the perturbation theory at higher temperatures than the Kondo temperature and is characterized by a heavyfermion behavior at low temperatures. ${ }^{1}$ On the other hand, in Kondo compounds, there exists a strong competition between the Kondo effect, which tends to demagnetize the system, and magnetism which tends to yield a magnetic ordering at low temperatures. ${ }^{2,3}$ It results that some cerium Kondo compounds are nonmagnetic and are characterized by a heavy-fermion (or "Fermi-liquid") behavior at very low temperatures, while the other ones order magnetically, generally in antiferromagnetic order, and have a smaller heavyfermion character. Among the first class, compounds such as $\mathrm{CeAl}_{3}, \mathrm{CeCu}_{6}$, and $\mathrm{CeCu}_{2} \mathrm{Si}_{2}$ have enormous values of the magnetic susceptibility and electronic specific-heat constant, typically larger than $1 \mathrm{~J} / \mathrm{mole}^{2}$ and present a $T^{2}$ behavior for the magnetic resistivity at very low temperatures. In the second class, compounds such as $\mathrm{CeAl}_{2}, \mathrm{CeB}_{6}, \mathrm{CeAg}$, or $\mathrm{CeRh}_{2} \mathrm{Si}_{2}$ order magnetically, most of them in the antiferromagnetic order and only a few in the ferromagnetic order, and present a smaller, although still large, heavy-fermion behavior characterized by values of $\gamma$ typically of order 100 $\mathrm{mJ} / \mathrm{mole} \mathrm{K}^{2}$.

The competition between the Kondo effect and the Ruderman-Kittel-Kasuya-Yosida (RKKY) interaction has been previously described by the well known "Doniach diagram, ${ }^{, 4}$ which will be presented in the next section. We will show that the predicted behavior of the Néel temperature $T_{N}$ is well followed experimentally in several cerium Kondo compounds, while the deduced Kondo temperature has a behavior clearly different from that of the one impurity value. The purpose of the present paper is, therefore, to present a "revisited" version of the Doniach diagram. In Sec. III, we describe the model including short-range antiferromagnetic correlations in the Kondo lattice for the nonmagnetic case and finally, in Sec. IV, we apply our theoretical results to cerium Kondo compounds and in particular to the pressure dependence of their Kondo temperature.

\section{STATE OF THE PROBLEM: THE EXPERIMENTAL SITUATION}

Since the purpose of the present paper is to re-examine in detail the Doniach diagram shown in Fig. 1, we will first study its present status. Let us call $T_{K 0}$ the Kondo temperature for a single impurity and $T_{N 0}$ the Néel (or Curie in a few cases) temperature, as if there were no Kondo effect. For the exchange Hamiltonian $H=-J_{K} \vec{s}_{c} \cdot \vec{S}, T_{K 0}$ is proportional to $\exp \left(-1 /\left|J_{K} \rho\right|\right)$ and $T_{N 0}$ to $\left|J_{K} \rho\right|^{2}$, where $\rho$ is here the den-

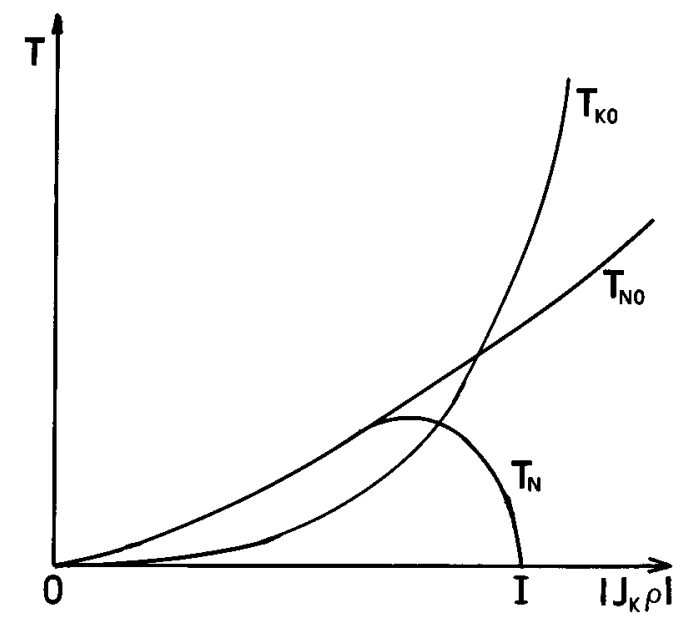

FIG. 1. Doniach diagram: Plot of the Néel and Kondo temperatures as a function of $\left|J_{K} \rho\right|$, as explained in text. 


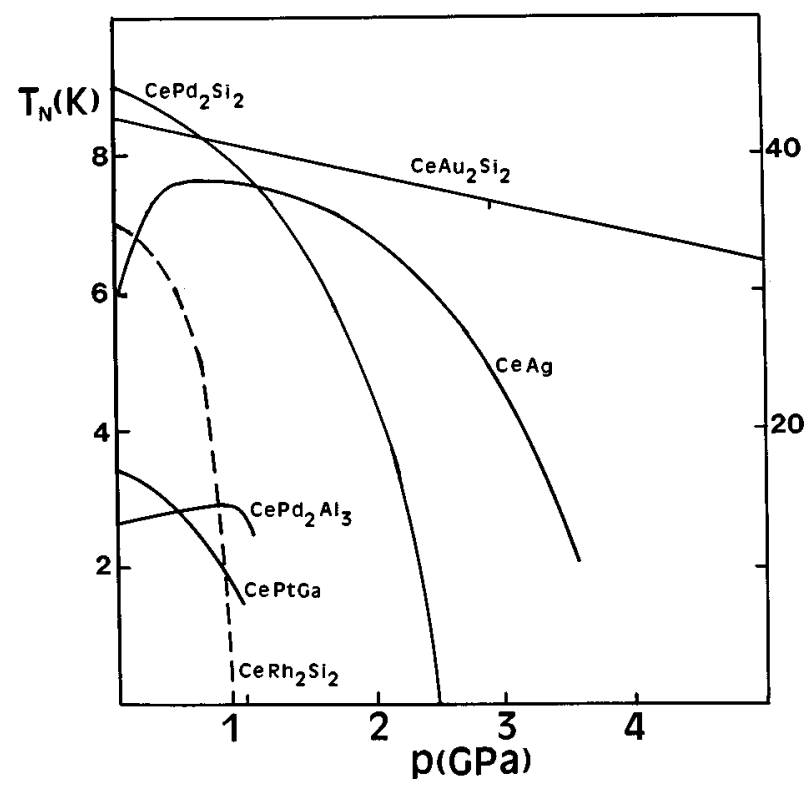

FIG. 2. Experimental curves of the pressure dependence of the Néel temperature for some cerium compounds. The full lines correspond to the left scale for the Néel temperature, while the dotted line of the $\mathrm{CeRh}_{2} \mathrm{Si}_{2}$ compound corresponds to the right scale.

sity of states for the conduction band at the Fermi energy. Thus, for small $\left|J_{K} \rho\right|$ values, $T_{N 0}$ is larger than $T_{K 0}$ and the system tends to order magnetically, frequently with a reduction of the magnetic moment due to the Kondo effect; ${ }^{5}$ on the contrary, for large $\left|J_{K} \rho\right|, T_{K 0}$ is larger than $T_{N 0}$ and the system tends to become nonmagnetic and does not order magnetically. The real ordering temperature $T_{N}$, therefore, increases initially with increasing $\left|J_{K} \rho\right|$, then passes through a maximum and tends to zero at a critical value $\left|J_{K} \rho\right|_{c}$ corresponding to point $\mathbf{I}$ of Fig. 1.

Such a behavior of $T_{N}$ has been experimentally observed with increasing pressure in several cerium Kondo compounds, namely $\mathrm{CeAl}_{2},{ }^{6,7} \quad \mathrm{CeAg},{ }^{8} \mathrm{CePd}_{2} \mathrm{Al}_{3},{ }^{9}$, $\mathrm{CePtGa},{ }^{10} \mathrm{CeIn}_{3},{ }^{11} \mathrm{CePd}_{2} \mathrm{Si}_{2},{ }^{12} \mathrm{CeAu}_{2} \mathrm{Si}_{2},{ }^{13} \mathrm{CeCu}_{2},{ }^{13}$ and $\mathrm{CeRh}_{2} \mathrm{Si}_{2} \cdot{ }^{14} \mathrm{~A}$ maximum has been observed in some of them, such as CeAg (Ref. 8) or $\mathrm{CePd}_{2} \mathrm{Al}_{3},{ }^{9}$ but in all these compounds, the Néel temperature decreases finally and tends to zero at high pressures, as shown in Fig. 2. A similar variation of $T_{N}$ has also been observed in $\mathrm{Ce}\left(\mathrm{Ru}_{x} \mathrm{Rh}_{1-x}\right){ }_{2} \mathrm{Si}_{2},{ }^{15}$ $\mathrm{CeSi}_{x},{ }^{16}$ or $\mathrm{CeNi}_{x} \mathrm{Pt}_{1-x},{ }^{17}$ with a variation of the relative concentration $x$. We will discuss in detail the first case later on, but the two other cases, namely $\mathrm{CeSi}_{x}$ (Ref. 16) or $\mathrm{CeNi}_{x} \mathrm{Pt}_{1-x},{ }^{17}$ are not really relevant for our present study since the experimentally observed transition to nonmagnetism corresponds probably here to a change of valence. ${ }^{18}$ This type of transition to an intermediate valence has been also observed in alloys such as $\mathrm{Ce}\left(\mathrm{Cu}_{x} \mathrm{Pd}_{1-x}\right)_{2} \mathrm{Si}_{2}$ (Ref. 19) or $\mathrm{Ce} M_{x} \mathrm{Pd}_{1-x}$ (with $M=\mathrm{Ni}$ or $\mathrm{Rh}$ ). ${ }^{20,21}$

In conclusion, the variation of the Néel temperature which is predicted by the Doniach diagram is well followed in many cerium Kondo compounds. However, the preceding description appears to be too much simplified for the real Kondo temperature $T_{K}$ which is experimentally observed in such cerium compounds. In fact, compounds with large $\left|J_{K} \rho\right|$ values have clearly much larger $\gamma$ values than those which have $\left|J_{K} \rho\right|$ values smaller than the critical value $\left|J_{K} \rho\right|_{c}$, as previously observed. ${ }^{2}$ From the proportionality relationship between $\gamma$ and $1 / T_{K}$, we could deduce that the corresponding $T_{K}$ values for the compounds with large $\left|J_{K} \rho\right|$ values must be much smaller than the $T_{K}$ values for compounds with small $\left|J_{K} \rho\right|$ values. For example, a previous analysis of the magnetic resistivity curves for both $\mathrm{CeAl}_{3}$ and $\mathrm{CeAl}_{2}$ compounds which are on the two sides of the magnetic-nonmagnetic transition, has given a value of 1.43 for the ratio of the corresponding $\left|J_{K} \rho\right|$ values; ${ }^{22}$ if then one takes simply the exponential dependence of the Kondo temperature according to the Doniach diagram, this would lead to a rapid increase of $T_{K}$ from $\mathrm{CeAl}_{2}$ to $\mathrm{CeAl}_{3}$, while the estimation of $T_{K}$ from the specific-heat results would give a $T_{K}$ roughly smaller by a factor 10 , for $\mathrm{CeAl}_{3}$, than that for $\mathrm{CeAl}_{2}$.

Recent measurements ${ }^{14}$ of the resistivity and specific heat under high pressure up to $17 \mathrm{kbar}$ at low temperatures have been performed in a $\mathrm{CeRh}_{2} \mathrm{Si}_{2}$ compound. At normal pressure, it orders antiferromagnetically with a relatively large value of the Néel temperature $T_{N}=35 \mathrm{~K}$ and a relatively small value of the electronic specific-heat constant $\gamma=22.8$ $\mathrm{mJ} / \mathrm{mole} \mathrm{K}^{2}$. The Néel temperature decreases rapidly with pressure and disappears at $9 \mathrm{kbar}$. On the other hand, the electronic specific-heat constant increases rapidly and goes through a broad maximum around $14 \mathrm{kbar}$ at a value of $\gamma=$ $80 \mathrm{~mJ} / \mathrm{mole}^{2}$, which is much larger than the normal pressure value. Thus, if we apply the proportionality relationship between $\gamma$ and $1 / T_{K}$, we obtain a clear decrease of $T_{K}$, by a factor of roughly 3 , in the pressure range where the compound changes from antiferromagnetic to nonmagnetic and then a slow increase of $T_{K}$ above $14 \mathrm{kbar}$. In fact, we must be careful in analyzing the variation of $T_{K}$ under pressure, because the proportionality relationship between $\gamma$ and $1 / T_{K}$ is valid only in the nonmagnetic case. But, above 9 kbar, the value of $T_{K}$ deduced from $\gamma$ is first decreasing and then increasing smoothly, yielding a rather smooth variation of $T_{K}$ above $9 \mathrm{kbar}$. On the other hand, resistivity measurements ${ }^{14}$ reveal a $T^{3}$ behavior at ambient and $7.3 \mathrm{kbar}$ pressure, but once antiferromagnetic order is suppressed, a Fermi-liquid $T^{2}$ dependence develops with a slope $A$ that decreases slowly. Taking $A$ proportional to $1 / T_{K}^{2}$, we obtain a very smooth increase of $T_{K}$ by a factor 1.3 between 12.4 and $17 \mathrm{kbar}$. Thus, there is, in $\mathrm{CeRh}_{2} \mathrm{Si}_{2}$ compound, an almost flat behavior of $T_{K}$ and it is clear that there is no exponential increase with pressure, as expected for the oneimpurity value $\mathrm{T}_{K 0}$ for the Kondo temperature.

Going back now to the case of $\mathrm{Ce}\left(\mathrm{Ru}_{1-x} \mathrm{Rh}_{x}\right)_{2} \mathrm{Si}_{2}$ (Ref. 15) alloys, it is well established that one goes from a nonmagnetic case for $x=0$ to a magnetically ordered case with increasing $x$ and the transition itself occurs at roughly $x=0.07$. Recent studies ${ }^{23}$ of these alloys have shown that the experimentally deduced Kondo temperature $T_{K}$ remains roughly constant and of order $20 \mathrm{~K}$ when the relative concentration $x$ is varying from $x=0$ to $x=0.2$, in the concentration range where the Néel temperature disappears; let us finally notice that cerium does not undergo any valence change in this concentration range, so that our present study will be applicable.

Thus, we will discuss in the following section a possible 
explanation for such a smaller value of $T_{K}$ compared to the value that could be deduced from the Doniach diagram for a given value of $\left|J_{K} \rho\right|$, by introducing short-range magnetic correlations in the Kondo lattice. ${ }^{24}$ Our calculation is performed in the "nonmagnetic regime,', where the proportionality relationship between $\gamma$ and $1 / T_{K}$ is surely well followed.

Moreover, the occurrence of short-range magnetic correlations has been experimentally observed by neutrondiffraction experiments at low temperatures in $\mathrm{CeCu}_{6}{ }^{25}$ $\mathrm{CeInCu}_{2},{ }^{26} \mathrm{CeRu}_{2} \mathrm{Si}_{2},{ }^{15,25,27}$ or $\mathrm{Ce}_{1-x} \mathrm{La}_{x} \mathrm{Ru}_{2} \mathrm{Si}_{2}$ (Refs. 15 and 27-29) alloys. It has been found that incommensurate and antiferromagnetic correlations develop at low temperatures below $T_{\text {cor }} \simeq 60-70 \mathrm{~K}$ in $\mathrm{CeRu}_{2} \mathrm{Si}_{2}$ or $T_{\text {cor }} \simeq 10 \mathrm{~K}$ in $\mathrm{CeCu}_{6}$, which are clearly larger than the Kondo temperature $T_{K} \simeq 14-23 \mathrm{~K}\left(\right.$ in $\mathrm{CeRu}_{2} \mathrm{Si}_{2}$ ) or $T_{K} \simeq 5 \mathrm{~K}$ (in $\mathrm{CeCu}_{6}$ ), for these two compounds which do not order magnetically at low temperatures. As we will see later on, one extra result of our calculation would be to compute the correlation temperature $T_{\text {cor }}$ in addition to the lattice Kondo temperature $T_{K}$ in the nonmagnetic domain.

\section{THE THEORETICAL MODEL}

We consider the following Hamiltonian to describe the Kondo lattice with short-range magnetic correlations:

$$
H=E_{0} \sum_{i \sigma} n_{i \sigma}^{f}+\sum_{k \sigma} \varepsilon_{k} n_{k \sigma}^{c}-J_{K} \sum_{i} \vec{s}_{i}^{c} \vec{S}_{i}^{f}-J_{H} \sum_{i, \delta} \vec{S}_{i}^{f} \vec{S}_{i+\delta}^{f},
$$

where $n_{i \sigma}^{f}=f_{i \sigma}^{*} f_{i \sigma}, \quad n_{k \sigma}^{c}=c_{k \sigma}^{*} c_{k \sigma}, \quad \vec{S}_{i}^{f}$, and $\vec{s}_{i}^{c}$ are, respectively, the number of $f$ electrons on site $i$ and spin $\sigma$, the number of conduction electrons $c$ for a wave vector $\vec{k}$ and spin $\sigma$, the spin for $f$ electrons on site $i$, and that for $c$ electrons. In Eq. (1), we take a zero width $f$ band, a conduction band with a width $2 W$ and a constant density of states. The third term is the $s-f$ exchange term and $J_{K}(<0)$ is the Kondo coupling. Here, we add to the regular Kondo lattice the last term of Eq. (1) which describes an Heisenberg interaction between neighboring $f$ magnetic moments; in the last term of Eq. (1), $i+\delta$ designs the nearest neighbors of atom $i$. We take here either $J_{H}>0$ for a ferromagnetic coupling or $J_{H}<0$ for an antiferromagnetic one, but in fact we will mostly be interested by the case $J_{H}<0$. Also, we take here first $J_{K}$ and $J_{H}$ independent from each other, although in fact it is not the case, since $J_{H}$ is proportional to $\rho\left|J_{K}\right|^{2}$ in the classical RKKY interaction, where $\rho$ is the density of states for the conduction band; finally in the last section, we will assume a relationship between $J_{H}$ and $J_{K}$ in order to derive a "revisited" version of the Doniach diagram.

We treat here the Hamiltonian (1) in a "mean-field" approximation similar to that used by Coleman and Andrei. ${ }^{30}$ This approximation allows us to find simultaneously the Kondo effect ${ }^{31}$ and the antiferromagnetic correlations in a method analogous to that of the resonating valence bond (RVB) introduced by Anderson. ${ }^{32}$ Thus, we introduce the two following mean values for the nonmagnetic state, namely,

$$
\lambda_{i \sigma}=\left\langle c_{i \sigma}^{*} f_{i \sigma}\right\rangle=\left\langle f_{i \sigma}^{*} c_{i \sigma}\right\rangle,
$$

which describes the Kondo formation of the singlet state between the $f$ and conduction electrons, and

$$
\Gamma_{i, i+\delta}=\left\langle f_{i \sigma}^{*} f_{i+\delta, \sigma}\right\rangle=\left\langle f_{i+\delta, \sigma}^{*} f_{i \sigma}\right\rangle,
$$

which describes the magnetic correlations between nearest neighbors, as in the RVB approach.

The Hamiltonian (1) can, therefore, be written in the mean-field approximation:

$$
\begin{aligned}
H= & E_{0} \sum_{i \sigma} n_{i \sigma}^{f}+\sum_{k \sigma} \varepsilon_{k} c_{k \sigma}^{*} c_{k \sigma}-J_{K} \sum_{i \sigma} \lambda_{i}\left(c_{i \sigma}^{*} f_{i \sigma}+f_{i \sigma}^{*} c_{i \sigma}\right) \\
& +J_{H} \sum_{i, \delta, \sigma}\left(\Gamma_{i, i+\delta} f_{i+\delta, \sigma}^{*} f_{i \sigma}+\Gamma_{i+\delta, i} f_{i \sigma}^{*} f_{i+\delta, \sigma}\right) \\
& -2 J_{K} \sum_{i} \lambda_{i}^{2}-J_{H} \sum_{i, \delta} \Gamma_{i, i+\delta} \Gamma_{i+\delta, i}
\end{aligned}
$$

We consider here only spin independent and translation invariant solutions for which we have $\lambda_{i, \sigma}=\lambda$ and $\Gamma_{i, i+\delta, \sigma}=\Gamma$. Then, we describe the conduction band in the tight-binding approximation and write

$$
\varepsilon_{k}=\frac{W}{z} \sum_{R} \cos k \cdot R,
$$

where $z$ is the number of neighbors of a given atom. Thus, the Heisenberg term of the Hamiltonian (4) can be written as

$$
J_{H} \Gamma \sum_{k, R} \cos k \cdot R f_{k, \sigma}^{*} f_{k, \sigma}=\frac{z J_{H} \Gamma}{W} \sum_{k} \varepsilon_{k} f_{k, \sigma}^{*} f_{f, \sigma} .
$$

This means that the magnetic interaction $J_{H}$ leads to a finite bandwidth for the $f$ states, while the Kondo interaction $J_{K}$ produces a hybridization between the conduction band of width $2 W$ and the $f$ band of effective width $2 B W$, with $B$ given by

$$
B=\frac{z J_{H} \Gamma}{W}
$$

The Hamiltonian (4) is easily diagonalized and the resulting eigenvalues are given by

$$
E^{ \pm}(k)=\frac{1}{2}\left[\varepsilon_{k}(1+B)+E_{0}\right] \pm \sqrt{\left[\varepsilon_{k}(1-B)-E_{0}\right]^{2}+4 J_{k}^{2} \lambda^{2}} .
$$

The position $E_{0}$ of the starting $f$ energy and the Fermi energy $E_{F}$ have to be determined self-consistently in order to obtain

$$
\begin{aligned}
& \left\langle\sum_{\sigma} f_{i \sigma}^{*} f_{i \sigma}\right\rangle=1, \\
& \left\langle\sum_{\sigma} c_{i \sigma}^{*} c_{i \sigma}\right\rangle=n .
\end{aligned}
$$

Thus, within this special mean-field approach, we have to deal with two hybridized bands of energies $E^{ \pm}(k)$ given by Eq. (8) and, using the two conditions (9), we have to deter- 
mine the two quantities $\lambda$ and $\Gamma$ as a function of temperature. Then, when $\lambda$ becomes equal to zero, the correlation function $\left\langle\vec{s}_{i}^{c} \vec{S}_{i}^{f}\right\rangle$ becomes also zero in our mean-field approximation; the temperature at which $\lambda$ becomes equal to zero corresponds, therefore, to the real Kondo temperature, $T_{K}$. Similarly, the temperature $T_{\text {cor }}$ at which $\Gamma$ becomes zero corresponds well to the temperature at which short-range magnetic correlations $\left\langle\vec{S}_{i}^{f} \vec{S}_{i+1}^{f}\right\rangle$ disappear.

Finally, the mean-field parameters $\lambda$ and $\Gamma$ are obtained by minimizing the total energy

$$
\begin{aligned}
E= & 2 \sum_{\vec{k}}\left[E^{+}(\vec{k}) f\left(E^{+}(\vec{k})\right)+E^{-}(\vec{k}) f\left(E^{-}(\vec{k})\right)\right]-z J_{H} \Gamma^{2} \\
& -2 J_{K} \lambda^{2},
\end{aligned}
$$

where the summation is made over all the $\vec{k}$ states and $f(E)$ is the Fermi-Dirac function.

\section{THE THEORETICAL RESULTS FOR THE SYMMETRIC CASE AND THE COMPARISON WITH EXPERIMENTS}

We will now present the results obtained for the considered nonmagnetic case, so that we cannot deduce the variation of the Néel temperature here, but just the temperature $T_{\text {cor }}$, related to the short-range magnetic correlations. Moreover, we restrict ourselves to the case of a half-filled conduction band $(n=1)$ where $E_{0}$ and the Fermi energy $E_{F}$ are consequently equal to each other; we set here $E_{0}=E_{F}=0$. The situation is symmetric with respect to $E_{0}=0$. The total resulting density of states presents a gap around the zero energy; it is very large and mostly of $f$ character on the two sides of the gap. Then, the total density of states decreases rapidly when one goes further from the zero value and finally reaches the constant value assumed for the conduction band.

Thus, we will present here successively some analytical results which have been obtained at $T=0,{ }^{24}$ and then the calculations performed at finite temperatures. ${ }^{33}$ Finally, we will present the $J_{K}$ dependence of $T_{K}$ and $T_{\text {cor }}$ when a realistic relationship between $J_{K}$ and $J_{H}$ is taken into account.

At $T=0$, analytical calculations can be performed and the results depend on the relative values of $\lambda$ and $\Gamma$. Three different cases should be considered and the shape of the two hybridized bands strongly depends on the parameters, as previously explained in Ref. 24 . We will discuss here only the case of small values of $J_{H} / J_{K}$ and more precisely the expressions given below are valid when $\left|J_{H} \Gamma\right|$ is smaller than $J_{K}^{2} \lambda^{2} / W$. Thus, we have firstly obtained for $\lambda$

$$
\lambda \approx\left(\frac{W(1-B)}{J_{K}}\right) \exp \left(\frac{W(1-B)}{J_{K}}\right),
$$

and we have also computed, in the same limit, $\Gamma$ and the energy $E$ of this solution

$$
\begin{gathered}
\Gamma \approx \frac{1}{4}+\frac{J_{K} \lambda^{2}}{W(1-B)}, \\
E=-\frac{W}{2}-\frac{J_{K}^{2} \lambda^{2}}{W(1-B)^{2}}+\frac{z J_{H}}{4}\left(\frac{1}{4}+\frac{J_{K}^{2} \lambda^{2}}{W^{2}(1-B)^{2}}\right) .
\end{gathered}
$$

Thus, the simple analytical results obtained at $T=0$ allow us to have first simple conclusions. According to Eq. (11), $\lambda$ has the same dependence as in earlier calculations without correlations, ${ }^{31}$ but the bandwidth is renormalized by the correlations: $W_{\text {eff }}=W(1-B)$. Since $\Gamma$ remains positive for reasonably small $J_{K} / W$ values, $W_{\text {eff }}$ can be larger or smaller than $W$ and consequently $\lambda$ can be decreased or increased depending on whether $J_{H}$ is negative or positive. Moreover, from the calculation of the energy (13) at $T=0$, we have found that the nonmagnetic phase is stabilized, i.e., that the Kondo state is more stable than the state without Kondo effect $(\lambda=0)$, when $J_{H}<0$.

Thus, if the exchange Heisenberg interaction is antiferromagnetic $\left(J_{H}<0\right), \lambda$ and consequently the real Kondo temperature $T_{K}$, as it will be explained later on, are reduced and the nonmagnetic Kondo state is stabilized by the antiferromagnetic short-range correlations. On the other hand, if $J_{H}$ is positive, the correlations increase $\lambda$ according to Eq. (11) and make the nonmagnetic phase less stable according to Eq. (13). In fact, the problem of ferromagnetic short-range correlations is more delicate, since the nonmagnetic phase is not the most stable one for $J_{H}>0$. In the following, we will discuss only the case of short-range antiferromagnetic correlations $\left(J_{H}<0\right)$ which decrease $\lambda$ and stabilize the nonmagnetic phase. However, the preceding theoretical results can account for the experimental fact that the heavy-fermion behavior is much more often observed in nearly antiferromagnetic systems than in nearly ferromagnetic ones.

Then, we have performed calculations at finite temperatures only in the case of antiferromagnetic correlations $\left(J_{H}<0\right)$, for the nonmagnetic solution and the half-filled conduction band $E_{0}=E_{F}=0$. The mean-field parameters $\lambda$ and $\Gamma$ are obtained by the minimization of the total energy given by Eq. (10). We have derived the $\lambda$ and $\Gamma$ curves as a function of temperature for different $J_{H}$ and $J_{K}$ values and the results depend essentially on the ratio $J_{K} / J_{H}$ rather than on the individual values of $J_{K}$ and $J_{H}$. Both the $\lambda$ and $\Gamma$ versus $\mathrm{T}$ curves start from a nonzero value, increase first, go through a maximum and decrease down to zero at a temperature corresponding to, respectively, $T_{K}$ and $T_{\text {cor }}$ in the present mean-field approximation, as previously explained. Let us finally make a short remark on the definition of $T_{K}$ in our mean-field approximation: in the one-impurity case, there are different forms for the prefactor before the exponential, depending on the used approximation or also eventually for example on the crystalline field splittings, but most of the $J_{K}$ dependence arises from the exponential itself. However, in the lattice case, there are not many available calculations and in our case, the prefactor does not depend on $J_{K}$; further work would be certainly very interesting to improve this description.

Figure 3 shows a typical plot of the two temperatures $T_{K}$ and $T_{\text {cor }}$ versus $J_{H}$ for a given large value $J_{K} / W=-1$. The Kondo temperature $T_{K}$, which is obviously equal to the oneimpurity Kondo temperature $T_{K 0}$ for $J_{H}=0$, remains constant for small $J_{H}$ values; after a very weak maximum, $T_{K}$ decreases slowly, indicating that the short-range antiferromagnetic correlations tend to decrease $T_{K}$. The temperature $T_{\text {cor }}$ remains equal to $T_{K}$ up to the $J_{H}$ value of the maximum of $T_{K}$ and then $T_{\text {cor }}$ increases linearly with $J_{H}$ according to 


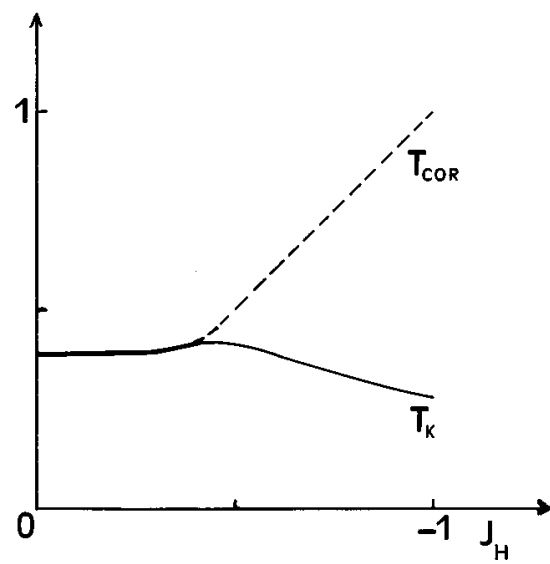

FIG. 3. Plot of the Kondo temperature $T_{K}$ (full line) and the correlation temperature $T_{\text {cor }}$ (dashed line) versus $J_{H}$, for $J_{K}=-1$.

the very simple law $T_{\text {cor }}=J_{H}$, predicted in the region where $\lambda=0$ with a number of neighbors $z=6$. Thus, it is remarkable to notice that there are essentially two regions: $T_{\text {cor }}=T_{K}$ for small $\left|J_{H}\right|$ values compared to $\left|J_{K}\right|$ and $T_{\text {cor }}>T_{K}$ when the effect of antiferromagnetic correlations overcomes the Kondo effect. A similar effect had been obtained in the Monte Carlo study of the two-impurity Kondo Hamiltonian. $^{34}$

But $J_{K}$ and $J_{H}$ cannot be considered as independent from each other and it is necessary to take into account the relationship between them to obtain the real dependence of the Kondo temperature $T_{K}$ versus $\left|J_{K}\right|$. The exchange $s$ - $f$ Hamiltonian itself yields both the RKKY interaction with an interaction going as $\left|J_{K}\right|^{2}$ and the Kondo effect, but we cannot derive together these two effects with only the $s-f$ Hamiltonian and that is the reason for introducing both the $s-f$ exchange and Heisenberg terms in the Hamiltonian (1). Since the $f$ - $f$ interaction term $J_{H}$ comes mostly from the indirect RKKY interaction, we take in the following:

$$
J_{H}=-\alpha\left|J_{K}\right|^{2}
$$

with a positive $\alpha$ value for the antiferromagnetic correlations.

Figures 4 and 5 give plots of the Kondo temperature $T_{K}$ and the correlation temperature $T_{\text {cor }}$ as a function of $J_{K}$ for a small value $\alpha=0.25$ and a large value $\alpha=2$ of the $\alpha$ parameter. We have plotted for comparison the nonperturbed (obtained for $J_{H}=0$ ) Kondo temperature $T_{k 0}$.

For the small value $\alpha=0.25$, the three temperatures $T_{K}, T_{K 0}$, and $T_{\text {cor }}$ are equal to each other, since the $f$ - $f$ correlations have almost no real effect. On the other hand, for $\alpha=2$, we see that the real Kondo temperature $T_{K}$ is first increasing with $\left|J_{K}\right|$ and almost equal to $T_{K 0}$, goes through a maximum, and decreases for large $\left|J_{K}\right|$ values. On the other hand, the correlation temperature $T_{\text {cor }}$ is much larger than $T_{K}$ and increases rapidly with $\left|J_{K}\right|$. Thus, for a large $\alpha$ value, both $T_{K}$ and $T_{\text {cor }}$ depart strongly from the $T_{K 0}$ curve.

Thus, according to Fig. 5, strong antiferromagnetic shortrange correlations decrease drastically the Kondo temperature with respect to that defined for one impurity and yield roughly constant values of $T_{K}$ versus $\left|J_{K}\right|$. Since pressure makes $\left|J_{K}\right|$ increase, the preceding theoretical result accounts

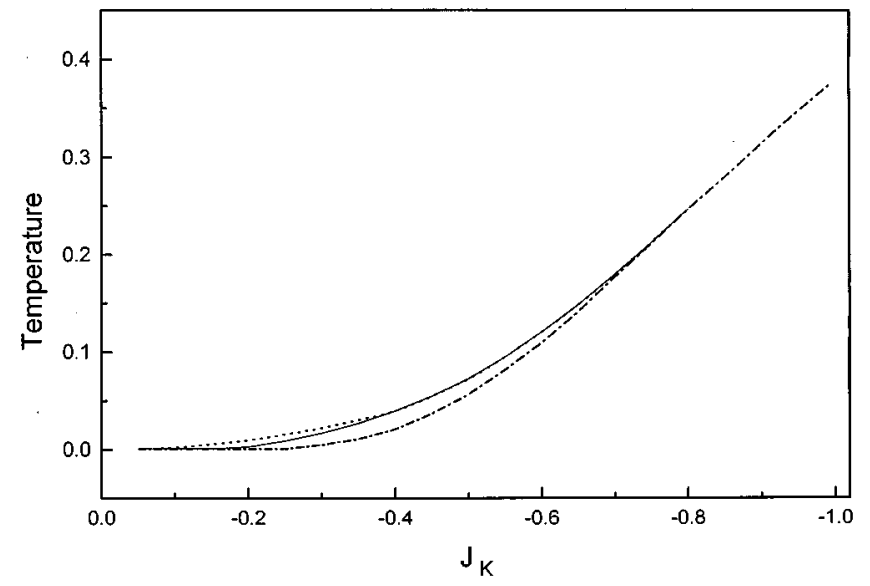

FIG. 4. Plot of the one-impurity Kondo temperature $T_{k 0}$ (dashed-dotted line), the real Kondo temperature $T_{K}$ (full line), and the correlation temperature $T_{\text {cor }}$ (dashed line) as a function of $J_{K}$ using formula (14) with $\alpha=0.25$.

for the experimentally observed rather constant values of $T_{K}$ observed in $\mathrm{CeRh}_{2} \mathrm{Si}_{2}$ (Ref. 14) compound under pressure when there is no longer any antiferromagnetic order. The similar behavior observed in $\mathrm{Ce}\left(\mathrm{Ru}_{1-x} \mathrm{Rh}_{x}\right){ }_{2} \mathrm{Si}_{2}$ (Ref. 15) alloys can also be accounted for by the present model. Resistivity measurements under pressure in $\mathrm{CeAu}_{2} \mathrm{Si}_{2}$ (Ref. 13) compound seem to yield also a similar behavior.

On the other hand, Fig. 3 gives a clear explanation of the occurrence of short-range antiferromagneticlike correlations in $\mathrm{CeRu}_{2} \mathrm{Si}_{2}$ or $\mathrm{CeCu}_{6}$ compounds at a temperature clearly larger than $T_{K}$. These compounds correspond to quite large $J_{H}$ values, while other compounds corresponding to smaller $J_{H}$ values would have equal $T_{K}$ and $T_{\text {cor }}$ values.

Thus, Fig. 5 provides, therefore, a "revisited" version of the Doniach diagram, but only for the nonmagnetic region above the instability point $\mathbf{I}$ where the Néel temperature $T_{N}$ disappears: The real Kondo temperature $T_{K}$ as defined for the lattice departs strongly from the one-impurity value and remains roughly constant with $\left|J_{K}\right|$ and moreover, we can add in this diagram a temperature $T_{\text {cor }}$ corresponding to the

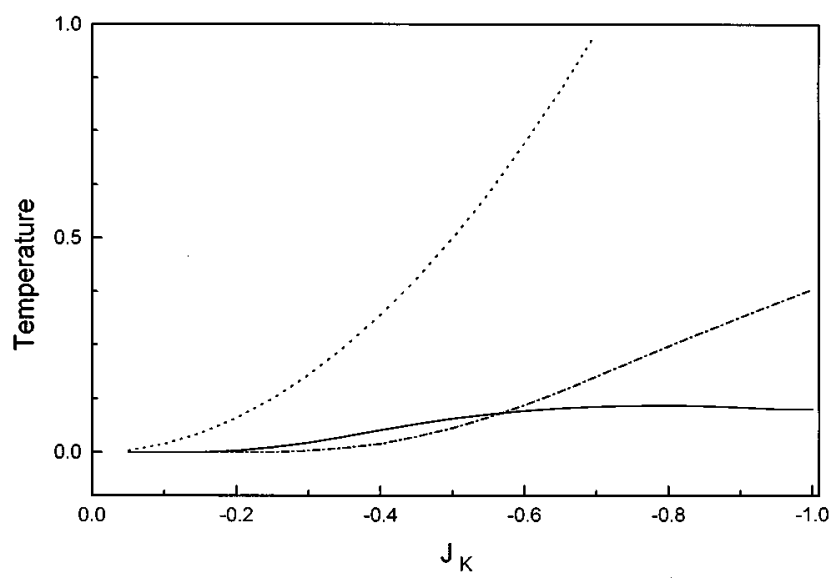

FIG. 5. Plot of the one-impurity Kondo temperature $T_{k 0}$ (dashed-dotted line), the real Kondo temperature $T_{K}$ (full line), and the correlation temperature $T_{\text {cor }}$ (dashed line) as a function of $J_{K}$ using formula (14) with $\alpha=2$. 
appearance of short-range correlations. These two defined temperatures can be observed experimentally in cerium compounds. Indeed, a full calculation describing completely the $J_{K}$ dependence of both $T_{K}$ and $T_{N}$ would be necessary to have a complete description of the Doniach diagram.

\section{CONCLUDING REMARKS}

Thus, the three main conclusions of our model can be summarized as follows:

(i) We propose here a "revisited" version of the Doniach diagram and we show that the real Kondo temperature $T_{K}$ for the lattice is roughly constant with $\left|J_{K}\right|$ or under pressure in the nonmagnetic region above the instability point $\mathbf{I}$ where the Néel temperature $T_{N}$ disappears. We can also understand qualitatively why the electronic specific-heat constants are generally much larger for the nonmagnetic cerium compounds than for the magnetically ordered ones.

(ii) We can account for the occurrence of antiferromagneticlike short-range correlations in some cerium compounds such as $\mathrm{CeCu}_{6}$ at a temperature $T_{\text {cor }}$ clearly larger than $T_{K}$.

(iii) We can finally understand why the heavy-fermion behavior is much more often observed in antiferromagnetic (or nearly antiferromagnetic) systems than in ferromagnetic ones.

Our results can be related to the exhaustion problem of concentrated Kondo alloys or compounds. ${ }^{35}$ In fact, for a very large Kondo coupling, the ground state is formed rather more by singlets between localized electron spins than by Kondo compensated spins. ${ }^{36}$ It could result in a decrease of the effective localized magnetic moment and then of the Kondo temperature itself.
The present theoretical derivation has been performed within a special mean-field approximation for the nonmagnetic case. The results are consequently valid in principle not too close to the instability point $\mathbf{I}$ of the Doniach diagram and the present model implies a Fermi-liquid behavior. However, a so-called "non-Fermi-liquid" behavior is often developing close to the I point and the behavior of the Kondo lattice near the magnetic instability is extensively studied from both theoretical and experimental points of view; in particular, a dense Kondo regime corresponding to a Fermiliquid behavior has also been obtained near $\mathbf{I}$ in the nonmagnetic regime at low temperatures with respect to the singleion Kondo temperature. ${ }^{37,38}$.

Experimental studies would be very interesting to perform in nearly antiferromagnetic systems, in order to observe this rather constant value of $T_{K}$ in a given range of $\left|J_{K}\right|$ in the nonmagnetic domain. It would also be interesting to check if the behavior of $T_{K}$ is really different in nearly ferromagnetic systems than in nearly antiferromagnetic ones.

Finally, further theoretical work including the study of the magnetic phase or the approach to magnetism from the nonmagnetic side would be necessary to develop, in order to get a full description of the Doniach diagram with both the Kondo and Néel temperatures.

\section{ACKNOWLEDGMENTS}

We acknowledge the support of Brazil-France cooperation agreement CAPES-COFECUB, Project No. 196/96. J.R.I. also acknowledges the support of the Brazilian agencies CNPq, FINEP, and FAPERGS.
${ }^{1}$ P. Fulde, J. Keller, and G. Zwicknagl, in Solid State Physics, edited by H. Ehrenreich and D. Turnbull (Academic, New York, 1988), Vol. 41, p. 1.

${ }^{2}$ B. Coqblin, J. Arispe, A. K. Bhattacharjee, and S. M. M. Evans, in Frontiers in Solid State Physics, edited by L. C. Gupta and M. S. Multani (World Scientific, Singapore, 1993), p. 75; S. M. M. Evans, A. K. Bhattacharjee, and B. Coqblin, Physica B 171, 293 (1991); C. Lacroix, J. Magn. Magn. Mater. 100, 90 (1991).

${ }^{3}$ B. Coqblin, J. Arispe, J. R. Iglesias, C. Lacroix, and Karyn Le Hur, J. Phys. Soc. Jpn. 65, Suppl. B 64 (1996).

${ }^{4}$ S. Doniach, Physica B 91, 231 (1977).

${ }^{5}$ J. G. Sereni, in Handbook on the Physics and Chemistry of Rare Earths, edited by K. A. Gschneidner, Jr. and L. Eyring (Elsevier Science B.V., Amsterdam, 1991), Vol. 15, p. 1.

${ }^{6}$ B. Barbara, H. Bartholin, D. Florence, M. F. Rossignol, and E. Walker, Physica B 86-88, 177 (1977).

${ }^{7}$ M. C. Croft, R. P. Guertin, L. C. Kupferberg, and R. D. Parks, Phys. Rev. B 20, 2073 (1979).

${ }^{8}$ A. Eiling and J. S. Schilling, Phys. Rev. Lett. 46, 364 (1981).

${ }^{9}$ F. Nolting, A. Eichler, S. A. M. Mentink, and J. A. Mydosh, Physica B 199-200, 614 (1994); S. A. M. Mentink, N. M. Bos, G. J. Nieuwenhuys, A. A. Menovsky, and J. A. Mydosh, ibid. 186-188, 497 (1993).

${ }^{10}$ Y. Uwatoko, T. Ishii, G. Oomi, and S. Malik, Physica B 206-207, 199 (1995).
${ }^{11}$ J. Flouquet, P. Haen, P. Lejay, P. Morin, D. Jaccard, J. Schweizer, C. Vettier, R. A. Fisher, and N. E. Phillips, J. Magn. Magn. Mater. 90-91, 377 (1990).

${ }^{12}$ P. Link and D. Jaccard, Physica B 223-224, 303 (1996).

${ }^{13}$ P. Link and D. Jaccard, Physica B 230-232, 31 (1997); D. Jaccard, P. Link, E. Vargoz, and K. Alami-Yadri, ibid. 230-232, 297 (1997).

${ }^{14}$ R. Movshovich, T. Graf, D. Mandrus, M. F. Hundley, J. D. Thompson, R. A. Fischer, N. E. Phillips, and J. L. Smith, Physica B 223-224, 126 (1996); T. Graf, J. D. Thompson, M. F. Hundley, R. Movshovich, Z. Fisk, D. Mandrus, R. A. Fischer, and N. E. Phillips, Phys. Rev. Lett. 78, 3769 (1997).

${ }^{15}$ J. Flouquet, S. Kambe, L. P. Regnault, P. Haen, J. P. Brison, F. Lapierre, and P. Lejay, Physica B 215, 77 (1995), and references therein.

${ }^{16}$ W. H. Lee, R. N. Shelton, S. K. Dhar, and K. A. Gschneidner, Jr., Phys. Rev. B 35, 8523 (1987).

${ }^{17}$ D. Gignoux and J. C. Gomez-Sal, Phys. Rev. B 30, 3967 (1984).

${ }^{18}$ J. G. Sereni, Physica B 206-207, 246 (1995).

${ }^{19}$ M. Weiden, O. Trovarelli, M. Gomez-Berisso, R. MüllerReisener, C. Geibel, and J. G. Sereni, Physica B 223-224, 299 (1996).

${ }^{20}$ J. G. Sereni, E. Beaurepaire, and J. P. Kappler, Phys. Rev. B 48, 3747 (1993).

${ }^{21}$ J. G. Sereni and J. P. Kappler, J. Phys.: Condens. Matter 6, 4771 (1994). 
${ }^{22}$ B. Cornut and B. Coqblin, Phys. Rev. B 5, 4541 (1972).

${ }^{23}$ Y. Miyako, T. Takeuchi, T. Taniguchi, S. Kawarazaki, K. Marumoto, R. Hamada, Y. Yamamoto, M. Ocio, P. Pari, and J. Hammann, J. Phys. Soc. Jpn. 65, Suppl. B 12, (1996).

${ }^{24}$ J. R. Iglesias, C. Lacroix, J. Arispe, and B. Coqblin, Physica B 223-224, 160 (1996).

${ }^{25}$ J. Rossat-Mignod, L. P. Regnault, J. L. Jacoud, C. Vettier, P. Lejay, J. Flouquet, E. Walker, D. Jaccard, and A. Amato, J. Magn. Magn. Mater. 76-77, 376 (1988).

${ }^{26}$ J. Pierre, P. Haen, C. Vettier, and S. Pujol, Physica B 163, 463 (1990).

${ }^{27}$ L. P. Regnault, W. A. C. Erkelens, J. Rossat-Mignod, P. Lejay, and J. Flouquet, Phys. Rev. B 38, 4481 (1988).

${ }^{28}$ J. M. Mignot, J. L. Jacoud, L. P. Regnault, J. Rossat-Mignod, P. Haen, P. Lejay, Ph. Boutrouille, B. Hennion, and D. Petitgrand, Physica B 163, 611 (1990).

${ }^{29}$ L. P. Regnault, J. L. Jacoud, J. M. Mignot, J. Rossat-Mignod, C.
Vettier, P. Lejay, and J. Flouquet, Physica B 163, 606 (1990).

${ }^{30}$ P. Coleman and N. Andrei, J. Phys.: Condens. Matter 1, 4057 (1989).

${ }^{31}$ C. Lacroix and M. Cyrot, Phys. Rev. B 20, 1969 (1979).

${ }^{32} \mathrm{P}$. W. Anderson, in Frontiers and Borderlines in Many Particle Physics, edited by R. Broglia and J. R. Schrieffer (NorthHolland, Amsterdam, 1988).

${ }^{33}$ C. Lacroix, J. R. Iglesias, J. Arispe, and B. Coqblin, Physica B 230-232, 503 (1997).

${ }^{34}$ R. M. Fye and J. E. Hirsch, Phys. Rev. B 40, 4780 (1989).

${ }^{35}$ P. Nozieres, Ann. Phys. (Paris) 10, 19 (1985).

${ }^{36}$ L. P. Gor'kov and Ju H. Kim, Philos. Mag. B 74, 447 (1996).

${ }^{37}$ M. A. Continentino, G. M. Japiassu, and A. Troper, Phys. Rev. B 39, 9734 (1989); M. A. Continentino, ibid. 47, 11587 (1993).

${ }^{38}$ A. Rosch, A. Schroder, O. Stockert, and H. v. Lohneysen, Phys. Rev. Lett. 79, 159 (1997). 